\title{
Response of micro- and macrovascular endothelial cells to starch-based fiber meshes for bone tissue engineering
}

\author{
Marina I. Santos ${ }^{\mathrm{a}, \mathrm{b}, \mathrm{c}}$, Sabine Fuchs ${ }^{\mathrm{c}}$, Manuela E. Gomes ${ }^{\mathrm{a}, \mathrm{b}}$, Ronald E. Unger ${ }^{\mathrm{c}}$, \\ Rui L. Reis ${ }^{\mathrm{a}, \mathrm{b}}$, C. James Kirkpatrick ${ }^{\mathrm{c}, *}$ \\ a3B's Research Group-Biomaterials, Biodegradables and Biomimetics, University of Minho, Campus de Gualtar, 4710-057 Braga, Portugal \\ ${ }^{\mathrm{b}}$ Department of Polymer Engineering, University of Minho, Campus de Azurém, 4800-058 Guimarães, Portugal \\ ${ }^{\mathrm{c}}$ Institute of Pathology, Johannes Gutenberg University Mainz, Langenbeckstr. 1, Mainz 55101, Germany
}

Received 1 May 2006; accepted 2 August 2006

Available online 1 September 2006

\begin{abstract}
The establishment of a functional vasculature is as yet an unrealized milestone in bone reconstruction therapy. For this study, fibermesh scaffolds obtained from a blend of starch and poly(caprolactone) (SPCL), that have previously been shown to be an excellent material for the proliferation and differentiation of bone marrow cells and thereby represent great potential as constructs for bone regeneration, were examined for endothelial cell (EC) compatibility. To be successfully applied in vivo, this tissue engineered construct should also be able to support the growth of ECs in order to facilitate vascularization and therefore assure the viability of the construct upon implantation. The main goal of this study was to examine the interactions between ECs and SPCL fiber meshes. Primary cultures of HUVEC cells were selected as a model of macrovascular cells and the cell line HPMEC-ST1.6R as a model for microvascular ECs.

Both macro- and microvascular ECs adhered to SPCL fiber-mesh scaffolds and grew to cover much of the available surface area of the scaffold. In addition, ECs growing on the SPCL fibers exhibited a typical morphology, maintained important functional properties, such as the expression of the intercellular junction proteins, PECAM-1 and VE-cadherin, the expression of the most typical endothelial marker vWF and sensitivity to pro-inflammatory stimuli, as shown by induction of the expression of cell adhesion molecules (CAMs) by lipopolysaccharide (LPS). These data indicate that ECs growing on SPCL fiber-mesh scaffolds maintain a normal expression of ECspecific genes/proteins, indicating a cell compatibility and potential suitability of these scaffolds for the vascularization process in bone tissue engineering in vivo.
\end{abstract}

(C) 2006 Elsevier Ltd. All rights reserved.

Keywords: Vascularization; Endothelial cells; Starch-based scaffolds; Bone tissue engineering

\section{Introduction}

A critical obstacle in tissue engineering approaches based on the in vitro culture of cell-scaffold constructs prior to implantation is the ability to maintain large masses of living cells upon transfer from the in vitro culture conditions into the host [1]. In vivo most cells are no more than $100 \mu \mathrm{m}$ away from the nearest capillary, which serves to supply oxygen and nutrients, remove waste products and

\footnotetext{
*Corresponding author. Tel.: + 496131 177301; fax: +49613117477301 .

E-mail address: kirkpatrick@pathologie.klinik.uni-mainz.de (C.J. Kirkpatrick).
}

transport biochemical signals [2]. Insufficient vascularization results in hypoxic cell death of engineered tissues [3] and consequently in implant failure [4]. Considering that the infiltration of blood vessels into a macroporous scaffold is a process that occurs at a rate of $<1 \mathrm{~mm}$ per day and that it typically takes $1-2$ weeks for the vascular structure to complete the penetration into relatively thin ( $3 \mathrm{~mm}$ thick) scaffolds [1], the need for the development of new approaches to increase the rate or augment vascularization is evident.

In the particular case of bone grafts, the lack of a successful blood supply is implicated as one of the major factors responsible for implant failure. In bone, angiogenesis is a fundamental process for both osseous formation 
and repair [3]. Thus, for example, in intramembranous bone formation extensive vascularization is observed at the transition of pre-osteoblasts to osteoblasts [5]. In endochondral bone formation, an avascular cartilage template is replaced by highly vascularized bone tissue [6]. In the repair of fractures by callus production the formation of soft callus is accompanied by strong angiogenic activity [7]. Accordingly, strategies that enhance angiogenesis should have positive effects on bone repair [3]. Several approaches have emerged to solve the lack of vascularization in bone grafts, such as incorporation of angiogenic factors in the scaffold to stimulate the endogenous angiogenic response [8-10], deposition of an angiogenic extracellular matrix on the surface of the implant by a tumorigenic cell line $[11,12]$, vector delivery of genes encoding angiogenic factors [13-15], bulk culturing of endothelial cells (ECs) as a homogenous population [13] or combined with osteoblasts [16]. These approaches all have in common the focus on ECs because these are the primary cells making up the vasculature.

Previous studies [17,18] have demonstrated that fiber meshes obtained from a blend of starch and polycaprolactone (SPCL) constitute an excellent scaffolding material for rat bone marrow stromal cells, allowing for their proliferation and differentiation into osteoblasts. Bioreactor studies have also shown the expression of an array of bone growth factors by marrow stromal cells growing on SPCL fiber-mesh scaffolds [19]. However, for a bone cellscaffold construct to be successful after implantation it should also elicit an adequate response of ECs. The scope of this work was to examine the ability of SPCL fiber meshes, a scaffold for bone repair, to serve as an appropriate substrate for ECs. For this purpose two types of ECs, human umbilical vein endothelial cell (HUVEC) and HPMEC-ST1, were cultured with SPCL fiber-mesh scaffolds and several functional and structural features of cells were analyzed such as viability, morphology, expression of EC markers and EC responsiveness to a proinflammatory stimulus.

\section{Materials and methods}

\subsection{Scaffolds}

The fiber-mesh scaffolds used in this study were based on SPLC (a polymeric blend of corn starch with polycaprolactone, $30 / 70 \mathrm{wt} \%$ ) and were obtained by a fiber bonding process, as described elsewhere [20]. The fiber-mesh scaffolds had a porosity of about $75 \%$ and for these experiments were cut into discs of approximately $8 \mathrm{~mm}$ diameter and $2 \mathrm{~mm}$ height. The scaffolds were sterilized by ethylene oxide and prior to cell seeding were immersed overnight in serum-free culture medium.

\subsection{Cells and culture conditions}

In this study, primary cultures of human ECs derived from umbilical vein (HUVEC) and the microvascular cell line HPMEC-ST1.6R developed from human pulmonary microvascular ECs were used. HUVECs were isolated from umbilical vein by collagenase digestion according to a published method [21]. HUVECs were cultured in M199 medium (Sigma-
Aldrich, Germany) supplemented with $10 \%$ fetal calf serum (FCS; Life Technologies, Germany), 10\% FCS (PAA Laboratories, Germany), $100 \mathrm{U} / 100 \mu \mathrm{g} / \mathrm{mL}$ Pen/Strep (Sigma-Aldrich, Germany), 2 mm glutamax I (Life Technologies, Germany), $25 \mu \mathrm{g} / \mathrm{mL}$ sodium heparin (Sigma-Aldrich, Germany) and $25 \mu \mathrm{g} / \mathrm{mL}$ endothelial cell growth supplement (ECGS, BD Biosciences, USA). Cells were used until the fourth passage. The HPMECST1.6R cell line was generated by transfection and displays the major constitutively expressed and inducible endothelial phenotypic markers [22]. HPMEC-ST1.6R was propagated in M199 culture medium supplemented with 20\% FCS (Life Technologies, Germany), $2 \mathrm{~mm}$ glutamax I, $100 \mathrm{U} / 100 \mu \mathrm{g} / \mathrm{mL}$ Pen/Strep (Sigma-Aldrich, Germany), $50 \mu \mathrm{g} / \mathrm{mL}$ sodium heparin, $25 \mu \mathrm{g} / \mathrm{mL}$ ECGS and $50 \mu \mathrm{g} / \mathrm{mL}$ geneticin 418 (Life Technologies, Germany) for selection of transfected cells. Both cells were cultured until confluence in culture flasks coated with $0.2 \%$ gelatin (Sigma-Aldrich, Germany).

\subsection{EC culture on SPCL fiber-mesh scaffolds}

SPCL fiber meshes were placed in 48-well plates and coated with $10 \mu \mathrm{g} /$ $\mathrm{mL}$ fibronectin in PBS (Roche, Germany) for $1 \mathrm{~h}$ at $37^{\circ} \mathrm{C}$. A control in PBS without fibronectin was performed under the same conditions. Confluent HPMEC-ST1.6R and HUVEC cells were trypsinized and a suspension of $1.5 \times 10^{5}$ HPMEC-ST1.6R cells or $2.5 \times 10^{5}$ HUVEC cells was added per scaffold. The culture plate was placed in the incubator for $2 \mathrm{~h}$ and then the cell-seeded SPCL fiber meshes were transferred to a new 24-well plate with $1.5 \mathrm{~mL}$ of fresh culture medium. Cells from the same donor grown on cell culture polystyrene were used as controls. The scaffolds were incubated under standard culture conditions $\left(37^{\circ} \mathrm{C}, 5 \%\right.$ $\mathrm{CO}_{2}$ ) for 3 and 7 days.

\subsection{EC imaging}

The viability and morphology of ECs on SPCL fiber meshes was assessed, after 3 and 7 days, by confocal laser scanning microscopy (CLSM) following calcein-AM staining and by scanning electron microscopy (SEM). For CLSM visualization, SPCL fiber meshes were incubated in culture medium with $0.1 \mu \mathrm{M}$ calcein-AM (Molecular Probes, Netherlands) for $10 \mathrm{~min}$. Calcein-AM is a non-fluorescent permeable compound that once inside viable cells is converted by intracellular esterases into a fluorescent cell impermeable form. The calcein-AMstained scaffold was placed on a microscope slide and observed by CLSM (Leica TCS NT). In order to examine the growth and morphology of ECs on SPCL fiber meshes, the samples were treated for SEM observation. Samples were fixed with $2 \%$ glutaraldehyde in $0.1 \mathrm{~m}$ sodium cacodylate buffer for $30 \mathrm{~min}$, postfixed in $1 \%$ osmium tetroxide for $1 \mathrm{~h}$, dehydrated in increasing concentrations of acetone, critical point dried and sputter coated with gold prior to SEM observation.

\subsection{DNA quantification}

The DNA content of each scaffold was measured using the PicoGreen DNA quantification assay (Molecular Probes). The samples were allowed to thaw at room temperature (RT) and then were sonicated for roughly 15 min. A description of the assay can be found elsewhere [23]. The number of cells on each scaffold was then calculated by correlation with the DNA of a known amount of ECs. Results are presented as means \pm standard deviation $(n=3)$.

\subsection{Immunostaining of EC markers}

The expression and localization of the EC markers PECAM-1, vWF and E-selectin was assessed by immunocytochemistry. The expression of PECAM-1 and vWF was assessed after growing HUVECs on SPCL fibermesh scaffolds for 7 days. The E-selectin staining was performed on HUVECs growing on SPCL fiber-mesh scaffolds and on cell culture plastic in the presence or absence of lipopolysaccharide (LPS). HUVEC 
cells on the scaffold and on plasticware were rinsed briefly with PBS and then fixed with $2 \%$ paraformaldehyde for $30 \mathrm{~min}$. Samples were then rinsed in PBS and treated with $0.1 \%$ Triton for $5 \mathrm{~min}$ at RT. After washing with PBS the samples were incubated for $45 \mathrm{~min}$ at RT with the primary antibodies: mouse anti-human PECAM-1 (1:50, Dako, Denmark), rabbit anti-human vWF (1:8000, Dako, Denmark) and mouse antihuman E-selectin (1:100, Monosan). Following PBS washing, a second incubation was performed for $45 \mathrm{~min}$ at RT with the secondary antibodies: anti-mouse Alexa Fluor 488 for PECAM and E-selectin staining and antirabbit Alexa Fluor 488 for vWF (Molecular Probes, The Netherlands). The nuclei were counterstained with $1 \mu \mathrm{g} / \mathrm{mL}$ Hoechst in PBS for $5 \mathrm{~min}$. SPCL fiber meshes were then washed with PBS, mounted with Gel/Mount (Natutec, Germany) and visualized by CLSM (Leica TCS SP2).

\subsection{Molecular analysis of pro-inflammatory genes}

For assessment of cell adhesion molecules (CAMs) expression on HUVEC seeded on to SPCL fiber meshes, these samples were cultured in the presence or absence of $1.0 \mu \mathrm{g} / \mathrm{mL}$ of LPS for $4 \mathrm{~h}$ (Sigma-Aldrich, Germany). HUVEC cells grown on plasticware with and without LPS were used as controls. The CAMs under analysis were E-selectin, ICAM, VCAM and the housekeeping gene $\beta$-actin was used as internal standard. Total RNA from HUVEC cells was extracted using the RNeasy Mini Kit (Qiagen, Germany) according to the manufacturer's protocol. Afterwards, the extracted RNA was reverse transcribed into cDNA, (Omniscript RT Kit, Qiagen) and used for polymerase chain reaction (PCR) analysis. Equal amounts of cDNA $(1 \mu \mathrm{g})$, measured by NanoDrop microspectrophotometer, were amplified by PCR with Taq DNA Polymerase Kit (Qiagen) and with gene-specific primer sets shown in Table 1. Thirty-five cycles were used for all genes, each one consisting of 2 min of denaturation at $94^{\circ} \mathrm{C}, 30 \mathrm{~s}$ of annealing (Table 1) and $30 \mathrm{~s}$ of chain elongation at $72^{\circ} \mathrm{C}$, followed by a final $10 \mathrm{~min}$ extension at $72{ }^{\circ} \mathrm{C}$. Amplification products were separated by electrophoresis on an agarose gel $(0.8 \%)$ and stained with ethidium bromide staining.

\section{Results}

\subsection{Micro- and macrovascular EC adhesion to SPCL fiber- mesh scaffolds}

ECs of micro- (HPMEC-ST1.6R) and macrovascular (HUVEC) origin were both able to attach and proliferate on fibronectin-coated SPCL fiber meshes (Fig. 1). However, in the absence of fibronectin coating, very few cells adhered, cells remained in a rounded-up shape and with time, no cells were detected on SPCL fiber meshes (data not shown). CLSM micrographs showed an increase in the cell number of HPMEC-ST1.6R (Fig. 1A and C) and HUVEC
(Fig. 1B and D) cells, on the surface of SPCL fiber meshes, between 3 and 7 days. Also, EC remained viable on SPCL fiber meshes as shown by their ability to convert calceinAM into a green fluorescent compound. To further confirm that cell numbers increased with time, cell DNA was isolated and quantified at two different time points, day 3 and 7 after addition of cells. As depicted in Fig. 1E, both HUVEC and HPMEC-ST1 cell numbers increased with time. Concerning cell morphology, SEM analysis showed that both micro- and macrovascular cells spread along the fibers, exhibited a typical flattened morphology and established contact with adjacent EC (Fig. 2).

\subsection{Immunohistochemistry of EC markers}

The expression of the EC markers vWF, PECAM and VE-cadherin was examined by immunohistochemistry. Immunostaining data showed vWF in a small dotted pattern surrounding the nuclei, and represented storage in Weibel-Palade bodies (Fig. 3A and B). Strong PECAM-1 staining was observed at the cell-cell interface typical of ECs on cell culture plastic and in vivo (Fig. 4A and B). Immunostaining of VE-cadherin exhibited labelling at the intercellular junctions between adjacent EC, similar to cells grown on cell culture plastic (data not shown). Thus, the labelling pattern and localization of the EC structural markers for cells growing on SPCL fiber-mesh materials exhibited a similar pattern to that observed for HUVEC cells grown on normal cell culture plastic (data not shown).

\subsection{Expression of pro-inflammatory genes}

ECs are involved in the inflammatory response in vivo through the expression of CAMs. These molecules are expressed by the inflamed endothelium in a sequential manner and in response to inflammatory stimuli such as cytokines and endotoxins [24]. Fig. 5 shows the mRNA expression of genes encoding CAMs of HUVECs grown on plastic wells and on SPCL fiber meshes, in the absence and in the presence of the pro-inflammatory stimulus. LPS, an endotoxin present in the cell wall of Gram-negative bacteria, was the selected stimulus. HUVEC grown on plastic wells in the absence of LPS expressed little or no

Table 1

Amplified genes, specific primer pair sequences and annealing temperature and product size

\begin{tabular}{llll}
\hline Name of gene (GenBank accession no.) & Product size (bp) & Annealing temperature $\left({ }^{\circ} \mathrm{C}\right)$ & Primer pair sequences \\
\hline$\beta$-actin (AB004047) & 574 & 65 & $\begin{array}{l}5^{\prime} \text {-AGCATTTGCGGTGGACGATGGAG-3' } \\
5^{\prime} \text {-GACCTGACTGACTACCTCATGA-3' }\end{array}$ \\
E-selectin (NM 000450) & 304 & 62 & $5^{\prime}$-ATCAACATGAGCTGCAGTGG-3' \\
ICAM (J03132) & 395 & 57 & $5^{\prime}$-AGCTTCCGTCTGATTCAAGG-3' \\
VCAM (X53051) & & $5^{\prime}$-TATTCAAACTGCCCTGATGG-3' \\
& 282 & 62 & $5^{\prime}$-CAGTGCGGCACGAGAAATTGG-3' \\
\hline
\end{tabular}



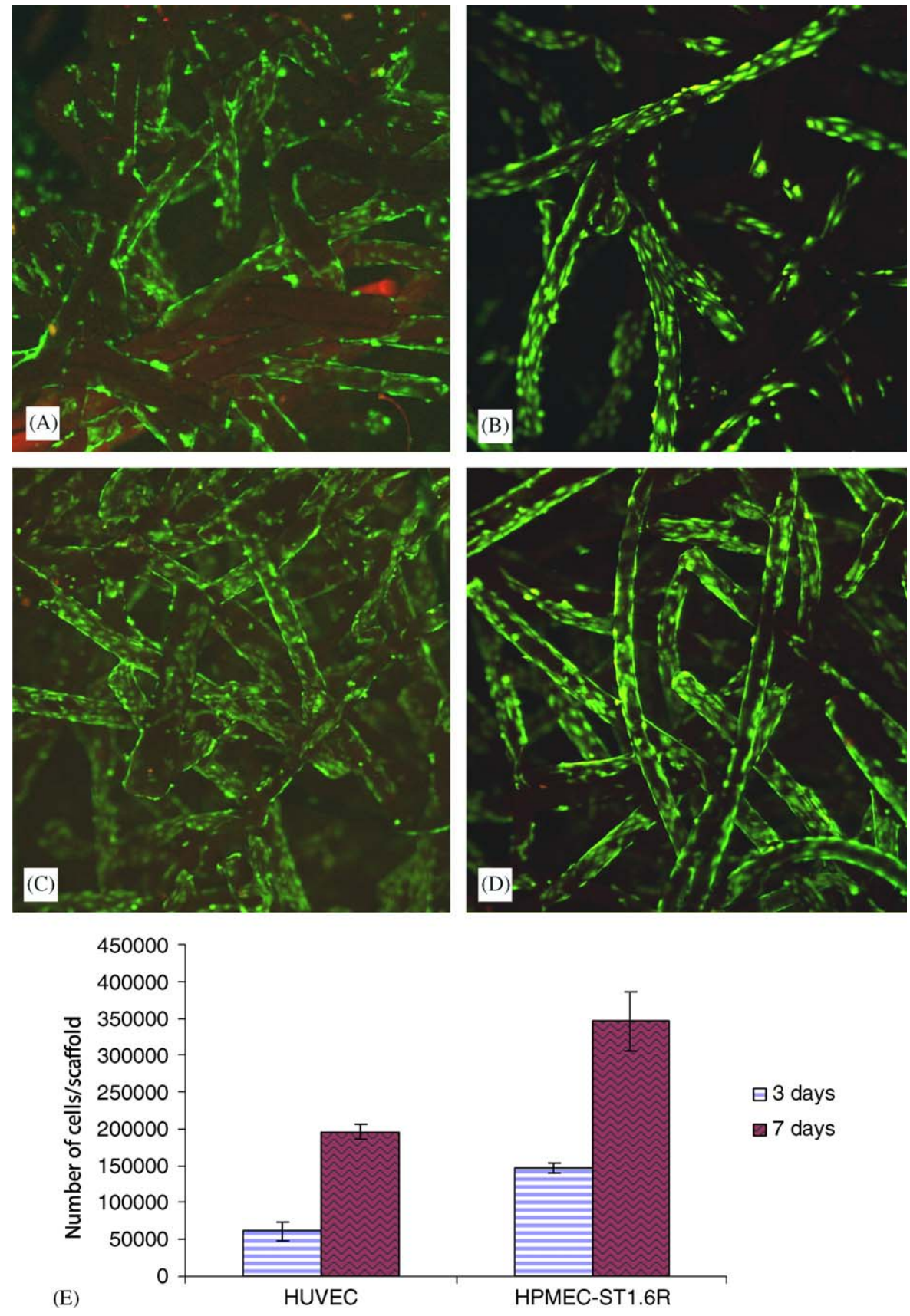

Fig. 1. Confocal micrographs of HPMEC-ST1.6R cells (A, C) and HUVEC (B, D) seeded on fibronectin-coated SPCL fiber meshes stained by calceinAM after 3 (A, B) and 7 days of culture (C, D). Magnification $(100 \times)$. Number of cells on SPCL fiber-mesh scaffolds after 3 and 7 days of culture based on DNA quantification (as described in Section 2) (E).

levels of the CAMs. However, in the presence of LPS an induction of CAMs expression was observed. Under noninflammatory conditions, HUVEC on the SPCL fiber-mesh scaffold had a low basal expression of ICAM, VCAM and E-selectin. In response to LPS, the expression of CAMs increased.

Since RT-PCR analysis examines the RNA of the entire population and does not give an indication of the gene expression at the single-cell level, we also carried out an E-selectin staining of cells growing on the SPCL fiber-mesh scaffolds in the presence and absence of LPS and compared the expression to the same cell type growing on normal cell culture plastic. As depicted in Fig. 6, a few HUVECs growing on both cell culture plastic and SPCL fiber meshes exhibited an expression of E-Selectin in the absence of LPS. We generally observe this for primary ECs in culture and between $1 \%$ and $5 \%$ of cells may exhibit expression of Eselectin in the absence of LPS stimulation (data not shown). However, after a $4 \mathrm{~h}$ stimulation with LPS, a large number of cells were observed on both materials exhibiting E-selectin expression (Fig. 6). Thus, ECs grown on the SPCL fiber meshes exhibited a similar pattern of expression 

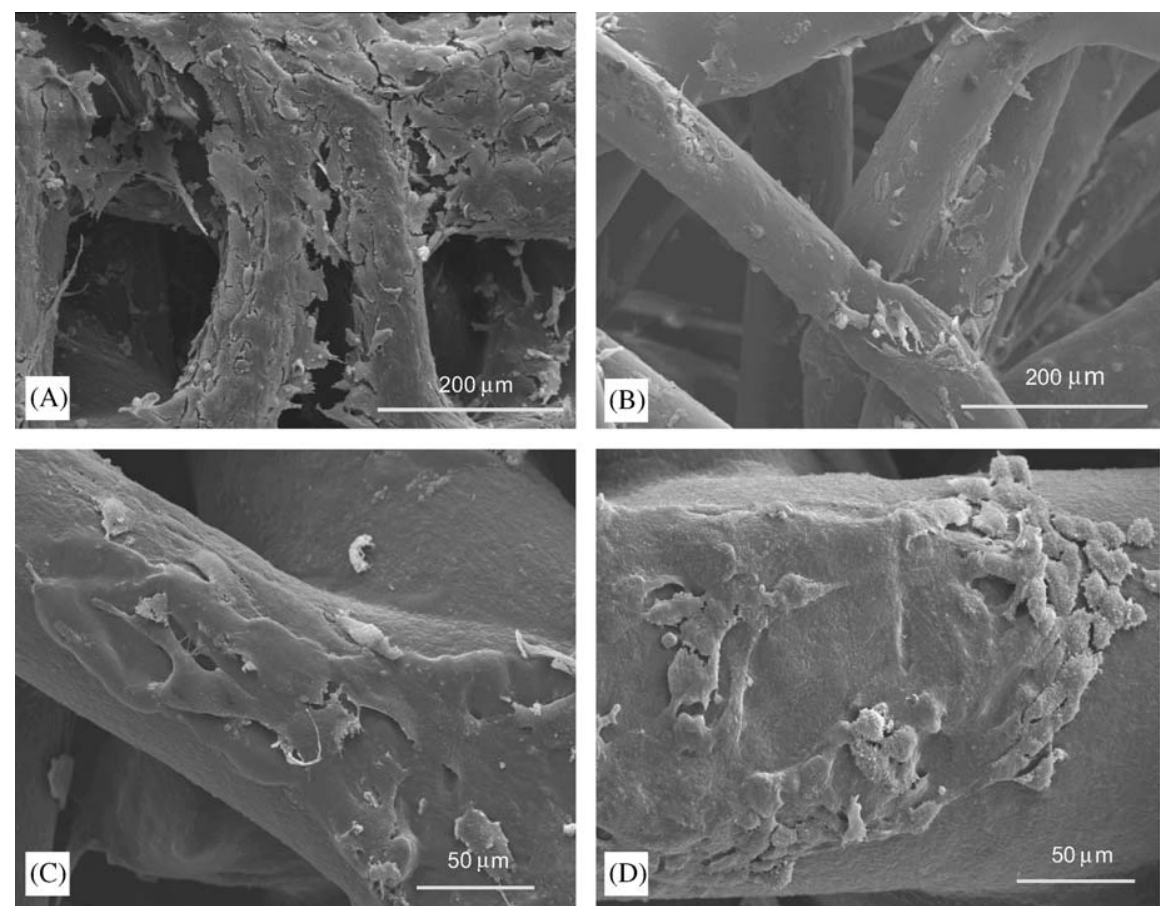

Fig. 2. SEM micrographs of HPMEC-ST1.6R (A, C) and HUVEC cells (B, D) on fibronectin-coated SPCL fiber meshes, after 3 (A, B) and 7 days of culture (C, D).
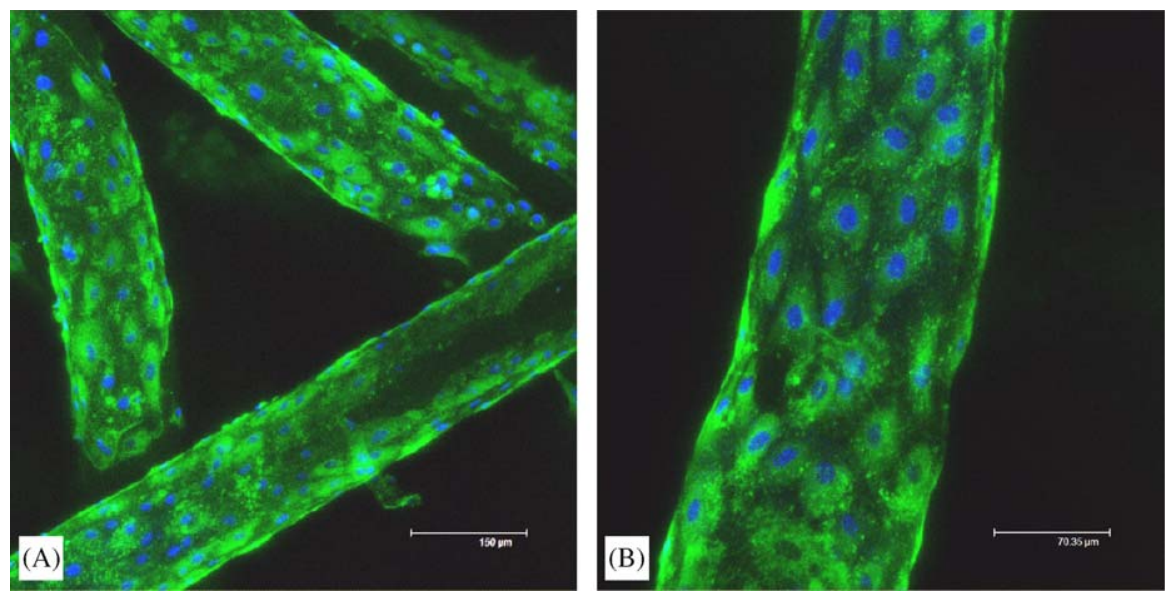

Fig. 3. Immunofluorescent micrographs of HUVEC cells grown for 1 week on fibronectin-coated SPCL fiber meshes and stained for vWF (green fluorescence) and with Hoechst for nuclear staining (blue fluorescence).

and induction of E-selectin compared to cells growing on plastic.

\section{Discussion}

After implantation of a biomaterial, a neovascularization process begins with the formation and outgrowth of microvasculature from the host tissue. For this reason, the ability of a tissue engineering scaffold to illicit an appropriate response from the host ECs is crucial for a successful vascularization of the implant. We have formerly described the SPCL fiber-mesh scaffold as a biomaterial for bone regeneration. Previous work has shown that this is an excellent scaffolding material for rat bone marrow stromal cells, allowing for their proliferation and differentiation into osteoblasts [17]. A successful implant not only requires the growth and function of the cells for a functioning tissue or organ replacement but also needs an intact vasculature to supply these cells with oxygen and nutrients and also to remove metabolites. Therefore, in this study, the growth, morphology and gene expression of human ECs on the SPCL fiber-mesh scaffolds were examined. HUVEC and the human microvascular EC line, HPMEC-ST1.6R, were used to assess EC interaction with the biomaterial since these cells maintain the EC phenotype in vitro and have been validated on many biomaterials [25-27].

Calcein-AM staining (Fig. 1) and SEM analysis (Fig. 2) of HPMEC-ST1 and HUVEC cells on SPCL fiber meshes 

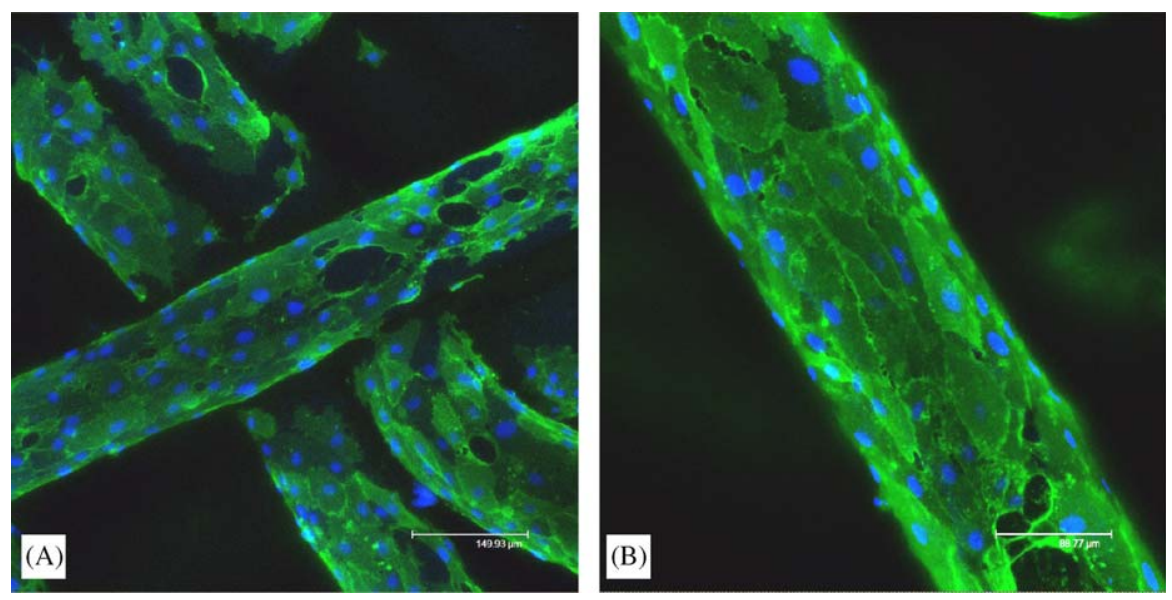

Fig. 4. Immunofluorescent micrographs of HUVEC cells grown for 1 week on fibronectin-coated SPCL fiber meshes and stained for PECAM-1 (green fluorescence) and with Hoechst for nuclear staining (blue fluorescence).

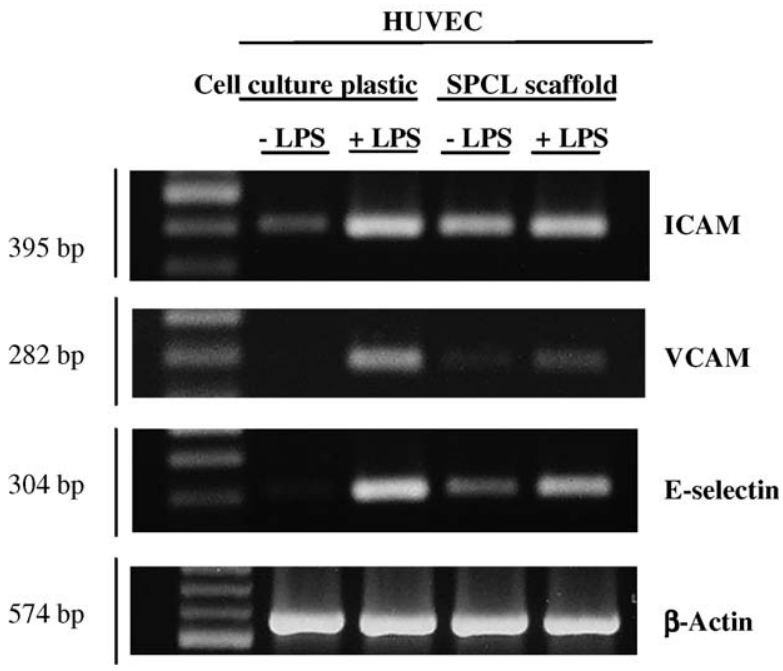

Fig. 5. PCR analysis of the genes that encode CAMs on HUVEC cells grown on SPCL fiber meshes for 7 days. CAM expression was assessed in the absence and presence of pro-inflammatory stimulus (LPS $1.0 \mu \mathrm{g} / \mathrm{m}$ for $4 \mathrm{~h}) . \beta$-actin was the selected housekeeping gene.

showed that with time these cells covered much of the available surface area of the fiber meshes. Cells grew to various depths in the fiber meshes and were also observed on the side opposite to that to which the cells were added (data not shown). An increase in cell number measured by DNA quantification was also observed for both cell types between day 3 and 7 (Fig. 1E). In addition, cells remained viable and retained the typical flattened morphology for the tested periods. However, this behavior was only shown on fibronectin-coated fiber meshes. This is not a surprising result, since it has been extensively reported in the literature $[28,29]$ that ECs show little adhesion and no proliferation on several kind of materials without prior coating with some form of extracellular matrix. Considering the relevance of the interactions of EC with extracellular matrix molecules for cell adhesion and proliferation [30,31], a common way to improve this behavior is accomplished by coating the material with cell adhesion proteins, such as fibronectin, prior to the cell seeding. Plasma treatment of the surface of SPCL fiber-mesh scaffold may also be a way to improve the adhesion of ECs without requiring the addition of extracellular matrix molecules [32] and we are currently examining this possibility. In addition to depending on the tight adhesion of the cells to the underlying basement membrane, the integrity of the endothelial layer is also strongly dependent on the junctions established between adjacent EC [33]. Such cell-cell adhesion is also crucial for vessels to sprout and the elongation process is mediated by a distinct series of cell surface receptors that includes PECAM-1 and VEcadherin [34]. PECAM-1, or platelet endothelial cell adhesion molecule-1, occurs on the EC membrane, close to the intercellular junctions, and regulates the adhesion of ECs to other cells of the same type and to leukocytes [35]. VE-cadherin is an adhesion molecule that mediates cell-cell contact between ECs and plays a relevant role in the maintenance of vascular integrity [36]. Immunocytochemical data revealed the typical localization of the EC markers vWF around the nuclei and PECAM-1 and VEcadherin at the intercellular junctions between adjacent cells (Figs. 3 and 4). The maintenance of the expression of PECAM-1, VE-cadherin and vWF, by HUVEC cells on SPCL fiber meshes is a good indicator of the interactions between EC and SPCL fiber meshes.

In addition to participating in angiogenesis, ECs also play an important role in the inflammatory response. During the inflammatory response to endotoxins or cytokines, a cross-talk between the endothelium and immune cells occurs resulting in the up-regulation of CAMs. These molecules are expressed on the inflamed endothelium in a sequential manner. These cells are involved in the steps leading to the adherence of circulating leukocytes from the blood flow and in their transmigration to the inflammatory focus. Thus, for example, E-selectin induces a prolonged contact between circulating leukocytes, resulting in a decelerated rolling along the endothelium [24]. VCAM-1 favors the adhesion and 

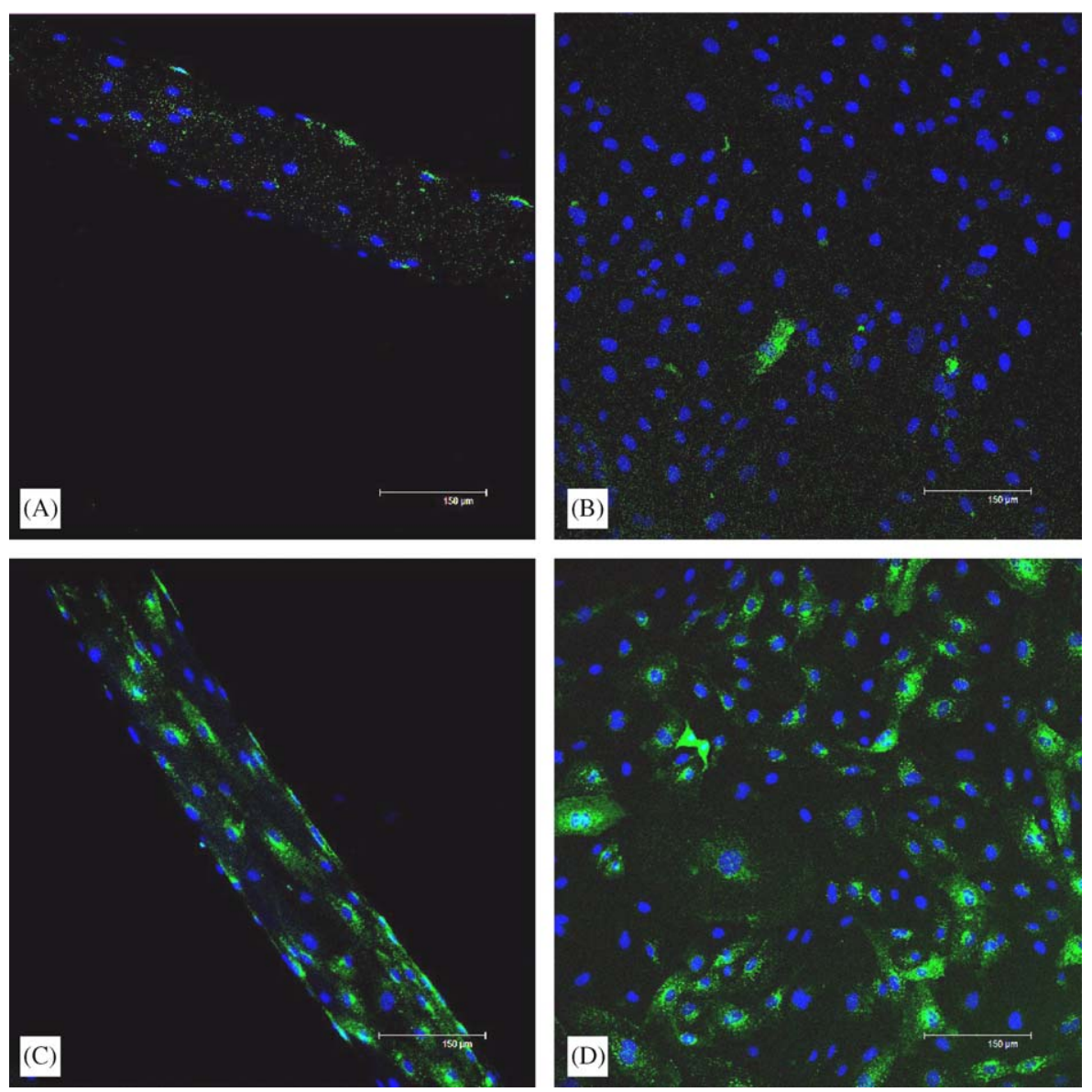

Fig. 6. Immunofluorescent images of E-selectin-stained HUVEC cells grown on fibronectin-coated SPCL fiber meshes (A and C) and on cell culture plastic (B and D) with and without LPS for $4 \mathrm{~h}$. A and B correspond to cells grown in the absence of LPS and C and D in its presence.

transendothelial migration especially of lymphocytes where they find the specific ligand [35]. ICAM-1 is constitutively expressed at a low level on EC and during inflammation is up-regulated several fold to facilitate EC-leukocyte adhesion, especially neutrophils and monocytes [37,38]. In contrast, E-selectin and VCAM are not usually expressed under physiological conditions, indicating that they require induction, a process involving de novo mRNA synthesis, resulting ultimately in the expression of the gene product, which then appears on the EC plasma membrane [38]. Analysis of expression of CAMs by RT-PCR and immunofluorescent staining of HUVEC cells grown on SPCL fiber meshes compared with HUVEC cells on plastic provided information regarding the ability of these cells to participate in the inflammatory response through the expression of CAMs in response to pro-inflammatory stimulus (Figs. 5 and 6). HUVEC cells growing on plastic wells were used as control and RT-PCR analysis of their mRNA revealed that little or none of the analyzed CAMs was expressed in unstimulated cells. In the presence of LPS the cells responded by rapidly inducing the synthesis of mRNA of ICAM, VCAM and E-selectin. The intensity of the bands for the three CAMs was higher than that detected in the absence of LPS. A similar result was observed by HUVEC growing on SPCL fiber meshes in the presence and absence of LPS. Little or no expression of E-selectin, VCAM and ICAM by HUVEC cells was observed in the absence of LPS, and in the presence of LPS, a clear increase was observed. This indicates that growth on SPCL fiber meshes does not affect the expression of the inflammatory genes, but after an inflammatorystimulating event, a normal induction of gene expression occurs. This was also confirmed through the immunofluorescent staining of the cells for E-selectin. Under noninflammatory conditions, only a few cells exhibited Eselectin staining. Upon LPS-stimulation, most of the cells exhibited some degree of E-selectin expression. Interestingly, the few cells expressing E-selectin in the absence of LPS also confirmed the results observed in the RT-PCR analysis (slight bands for E-selectin in the unstimulated cells, Fig. 5). We routinely see that up to $5 \%$ of freshly isolated human ECs may express CAMs (unpublished data). However, in all cases, after the addition of LPS, an increase in the expression of CAMs was observed, indicating normal cell behavior when growing on the SPCL fiber meshes.

\section{Conclusion}

It was found that endothelial cells (ECs) from both macro- and microvascular origin adhered to SPCL fiber 
meshes and grew over much of the surface area of the scaffold, with cell viability being maintained up to at least 7 days after addition to the scaffold. Moreover, SPCL fibermesh scaffolds supported the maintenance of EC morphological structure. Important functions such as endothelial integrity were maintained as shown by the expression of the endothelial intercellular junction proteins, PECAM-1 and VE-cadherin. The expression of the most typical endothelial marker vWF was also detected at single-cell level. Furthermore, ECs cultures onto SPCL fiber meshes were sensitive to a pro-inflammatory stimulus as was shown by the enhancement in the expression of CAMs induced by LPS. The results obtained demonstrate that SPCL fiber meshes are an excellent substrate for the growth of human ECs required for the vascularization process. Our findings, coupled with those previously reported for bone marrow cells, suggest that SPCL fiber meshes may have a potential for use as a scaffold material for bone tissue engineering applications.

\section{Acknowledgments}

M.I. Santos and M.E. Gomes would like to acknowledge the Portuguese Foundation for Science and Technology (FCT) for their PhD and Post-Doc scholarship (SFRH/ $\mathrm{BD} / 13428 / 2003$ and $\mathrm{SFRH} / \mathrm{BPD} / 20412 / 2004)$. This work was partially supported by FCT through funds from POCTI and/or FEDER programs and by the European Union-funded STREP Project HIPPOCRATES (NMP3CT-2003-505758). This work was carried out under the scope of the European NoE EXPERTISSUES (NMP3CT-2004-500283).

The authors would also like to acknowledge A. Sartoris, B. Malenica, M. Muller and. K. Molter for their excellent technical assistance.

\section{References}

[1] Nomi M, Atala A, Coppi PD, Soker S. Principals of neovascularization for tissue engineering. Mol Aspects Med 2002;23(6):463-83.

[2] Freed LE, Vunjak-Novakovic G. Culture of organized cell communities. Adv Drug Deliv Rev 1998;33(1-2):15-30.

[3] Stahl A, Wenger A, Weber H, Stark GB, Augustin HG, Finkenzeller G. Bi-directional cell contact-dependent regulation of gene expression between endothelial cells and osteoblasts in a three-dimensional spheroidal coculture model. Biochem Biophys Res Commun 2004; 322(2):684-92.

[4] Cassell OC, Hofer SO, Morrison WA, Knight KR. Vascularisation of tissue-engineered grafts: the regulation of angiogenesis in reconstructive surgery and in disease states. Br J Plast Surg 2002;55(8):603-10.

[5] Deckers MM, van Bezooijen RL, van der Horst G, Hoogendam J, van Der Bent C, Papapoulos SE, et al. Bone morphogenetic proteins stimulate angiogenesis through osteoblast-derived vascular endothelial growth factor A. Endocrinology 2002;143(4):1545-53.

[6] Maes C, Carmeliet P, Moermans K, Stockmans I, Smets N, Collen D, et al. Impaired angiogenesis and endochondral bone formation in mice lacking the vascular endothelial growth factor isoforms VEGF164 and VEGF188. Mech Dev 2002;111(1-2):61-73.

[7] Carano RA, Filvaroff EH. Angiogenesis and bone repair. Drug Discov Today 2003;8(21):980-9.
[8] Huang YC, Kaigler D, Rice KG, Krebsbach PH, Mooney DJ. Combined angiogenic and osteogenic factor delivery enhances bone marrow stromal cell-driven bone regeneration. J Bone Miner Res 2005;20(5):848-57.

[9] Kent Leach J, Kaigler D, Wang Z, Krebsbach PH, Mooney DJ. Coating of VEGF-releasing scaffolds with bioactive glass for angiogenesis and bone regeneration. Biomaterials 2006;27(17): 3249-55.

[10] Murphy WL, Simmons CA, Kaigler D, Mooney DJ. Bone regeneration via a mineral substrate and induced angiogenesis. J Dent Res 2004;83(3):204-10.

[11] Kidd KR, Nagle RB, Williams SK. Angiogenesis and neovascularization associated with extracellular matrix-modified porous implants. J Biomed Mater Res 2002;59(2):366-77.

[12] Kidd KR, Williams SK. Laminin-5-enriched extracellular matrix accelerates angiogenesis and neovascularization in association with ePTFE. J Biomed Mater Res A 2004;69(2):294-304.

[13] Moldovan NI, Ferrari M. Prospects for microtechnology and nanotechnology in bioengineering of replacement microvessels. Arch Pathol Lab Med 2002;126(3):320-4.

[14] Soker S, Machado M, Atala A. Systems for therapeutic angiogenesis in tissue engineering. World J Urol 2000;18(1):10-8.

[15] Alessandri G, Emanueli C, Madeddu P. Genetically engineered stem cell therapy for tissue regeneration. Ann N Y Acad Sci 2004;1015: 271-84.

[16] Wenger A, Stahl A, Weber H, Finkenzeller G, Augustin HG, Stark GB, et al. Modulation of in vitro angiogenesis in a three-dimensional spheroidal coculture model for bone tissue engineering. Tissue Eng 2004;10(9-10):1536-47.

[17] Gomes ME, Sikavitsas VI, Behravesh E, Reis RL, Mikos AG. Effect of flow perfusion on the osteogenic differentiation of bone marrow stromal cells cultured on starch-based three-dimensional scaffolds. J Biomed Mater Res 2003;67A(1):87-95.

[18] Tuzlakoglu K, Bolgen N, Salgado AJ, Gomes ME, Piskin E, Reis RL. Nano- and micro-fiber combined scaffolds: a new architecture for bone tissue engineering. J Mater Sci Mater Med 2005;16(12): 1099-104.

[19] Gomes ME, Bossano CM, Johnston CM, Reis RL, Mikos AG. In vitro localization of bone growth factors in constructs of biodegradable scaffolds seeded with marrow stromal cells and cultured in a flow perfusion bioreactor. Tissue Eng 2006;12(1):177-88.

[20] Gomes ME, Godinho JS, Tchalamov D, Cunha AM, Reis RL. Alternative tissue engineering scaffolds based on starch: processing methodologies, morphology, degradation and mechanical properties. Mater Sci Eng C-Biomimetic Supramol Syst 2002;20(1-2):19-26.

[21] Jaffe EA, Nachman RL, Becker CG, Minick CR. Culture of human endothelial cells derived from umbilical veins-identification by morphologic and immunological criteria. J Clin Invest 1973;52(11): 2745-56.

[22] Krump-Konvalinkova V, Bittinger F, Unger RE, Peters K, Lehr HA, Kirkpatrick CJ. Generation of human pulmonary microvascular endothelial cell lines. Lab Invest 2001;81(12):1717-27.

[23] Punshon G, Vara DS, Sales KM, Kidane AG, Salacinski HJ, Seifalian AM. Interactions between endothelial cells and a poly (carbonate-silsesquioxane-bridge-urea) urethane. Biomaterials 2005; 26(32):6271-9.

[24] Muller AM, Hermanns MI, Cronen C, Kirkpatrick CJ. Comparative study of adhesion molecule expression in cultured human macro- and microvascular endothelial cells. Exp Mol Pathol 2002;73(3):171-80.

[25] Unger RE, Huang Q, Peters K, Protzer D, Paul D, Kirkpatrick CJ. Growth of human cells on polyethersulfone (PES) hollow fiber membranes. Biomaterials 2005;26(14):1877-84.

[26] Unger RE, Peters K, Wolf M, Motta A, Migliaresi C, Kirkpatrick CJ. Endothelialization of a non-woven silk fibroin net for use in tissue engineering: growth and gene regulation of human endothelial cells. Biomaterials 2004;25(21):5137-46.

[27] Unger RE, Peters K, Huang Q, Funk A, Paul D, Kirkpatrick CJ. Vascularization and gene regulation of human endothelial cells 
growing on porous polyethersulfone (PES) hollow fiber membranes. Biomaterials 2005;26(17):3461-9.

[28] Chu CF, Lu A, Liszkowski M, Sipehia R. Enhanced growth of animal and human endothelial cells on biodegradable polymers. Biochim Biophys Acta 1999;1472(3):479-85.

[29] Boura C, Muller S, Vautier D, Dumas D, Schaaf P, Claude Voegel J, et al. Endothelial cell-interactions with polyelectrolyte multilayer films. Biomaterials 2005;26(22):4568-75.

[30] Pompe T, Markowski M, Werner C. Modulated fibronectin anchorage at polymer substrates controls angiogenesis. Tissue Eng 2004;10(5-6):841-8.

[31] Marin V, Kaplanski G, Gres S, Farnarier C, Bongrand P. Endothelial cell culture: protocol to obtain and cultivate human umbilical endothelial cells. J Immunol Methods 2001;254(1-2):183-90.

[32] Pu FR, Williams RL, Markkula TK, Hunt JA. Effects of plasma treated PET and PTFE on expression of adhesion molecules by human endothelial cells in vitro. Biomaterials 2002;23(11):2411-28.

[33] Ayalon O, Sabanai H, Lampugnani MG, Dejana E, Geiger B. Spatial and temporal relationships between cadherins and pecam-1 in cell-cell junctions of human endothelial-cells. J Cell Biol 1994;126(1):247-58.

[34] Cines DB, Pollak ES, Buck CA, Loscalzo J, Zimmerman GA, McEver RP, et al. Endothelial cells in physiology and in the pathophysiology of vascular disorders. Blood 1998;91(10): 3527-61.

[35] Cenni E, Granchi D, Ciapetti G, Verri E, Cavedagna D, Gamberini $\mathrm{S}$, et al. Expression of adhesion molecules on endothelial cells after contact with knitted Dacron. Biomaterials 1997;18(6):489-94.

[36] Nachtigal P, Gojova A, Semecky V. The role of epithelial and vascular-endothelial cadherin in the differentiation and maintenance of tissue integrity. Acta Med (Hradec Kralove) 2001;44(3):83-7.

[37] Remy M, Valli N, Brethes D, Labrugere C, Porte-Durrieu MC, Dobrova NB, et al. In vitro and in situ intercellular adhesion molecule-1 (ICAM-1) expression by endothelial cells lining a polyester fabric. Biomaterials 1999;20(3):241-51.

[38] Kirkpatrick CJ, Wagner M, Kohler H, Bittinger F, Otto M, Klein CL. The cell and molecular biological approach to biomaterial research: a perspective. J Mater Sci Mat Med 1997;8(3):131-41. 